\title{
ON MAPPING CONES OF SUSPENSION ELEMENTS OF FINITE ORDER IN THE HOMOTOPY GROUPS OF A WEDGE OF SPHERES
}

\author{
IMRE BOKOR
}

(Communicated by Frederick R. Cohen)

\begin{abstract}
The genus of the mapping cone $C_{f}$ of a map $f: S^{m-1} \rightarrow \bigvee S^{n}$ $(m>n>1)$ representing a suspension element of finite order in $\pi_{m-1}\left(\bigvee S^{n}\right)$ is classified by a subgroup $G_{f}$ of $\pi_{m-1}\left(S^{n}\right)$ depending only on the homotopy type of $C_{f}$. The group $G_{f}$ finds application in proving that the genus of $C_{f}$ is trivial whenever $C_{f}$ has sufficiently many $n$-cells, the number being limited by the torsion subgroup of $\pi_{m-1}\left(S^{n}\right)$.
\end{abstract}

The genus of the mapping cone $C_{f}$ of a map $f: S^{m-1} \rightarrow S^{n}$ representing a suspension element of finite order in $\pi_{m-1}\left(S^{n}\right)$ has been characterised both geometrically and algebraically as in [6]. (Recall-or see [2], for examplethat two spaces of the homotopy type of a nilpotent CW complex of finite type are said to be of the same genus if their $p$-localisations are homotopy equivalent at every prime $p$.)

Theorem 0.1 (cf. [6]). If $f, g: S^{m-1} \rightarrow S^{n}$ represent suspension elements of finite order in $\pi_{m-1}\left(S^{n}\right)$, then the following are equivalent:

(1) $C_{f}$ and $C_{g}$ are of the same genus.

(2) $C_{f} \vee S^{m} \vee S^{n}$ and $C_{g} \vee S^{m} \vee S^{n}$ are homotopy equivalent.

(3) Both $f$ and $g$ generate the same subgroup of $\pi_{m-1}\left(S^{n}\right)$.

Llerena [3] generalised the geometric condition (2) to the case of the mapping cone $C_{f}$ of $f: S^{m-1} \rightarrow \bigvee S^{n}$, where $f$ represents a suspension element of finite order in $\pi_{m-1}\left(\bigvee S^{n}\right)$. (Here we have abbreviated $\bigvee_{i=1}^{K} S^{n}$ to $\bigvee S^{n}$ and shall continue to do so except when there is danger of confusion.)

Theorem 0.2 (cf. [3]). If $f, g: S^{m-1} \rightarrow \bigvee S^{n} \quad(m>n>1)$ represent suspension elements of finite order in $\pi_{m-1}\left(\bigvee S^{n}\right)$, then the following are equivalent:

(1) $C_{f}$ and $C_{g}$ are of the same genus.

(2) $C_{f} \vee S^{m} \vee \bigvee S^{n}$ and $C_{g} \vee S^{m} \vee \vee S^{n}$ are homotopy equivalent.

Received by the editors May 7, 1991.

1991 Mathematics Subject Classification. Primary 55P15, 55P45.

Key words and phrases. CW complex, genus, suspension.

The author wishes to express his gratitude to the CRM of the Institut d'Estudis Catalans, Barcelona, for its support in the preparation of this paper. 
(3) There is a finite wedge of spheres, $W$, with $C_{f} \vee W$ homotopy equivalent to $C_{g} \vee W$.

The purpose of this note is to generalise the algebraic condition (3) of Theorem 0.1 and to derive some of its consequences. Specifically, we show how to assign to each map $f: S^{m-1} \rightarrow \bigvee S^{n}$ a subgroup, $G_{f}$, of $\pi_{m-1}\left(S^{n}\right)$ which classifies the genus of the mapping cone $C_{f}$. When $K=1$ the group $G_{f}$ is precisely the subgroup of $\pi_{m-1}\left(S^{n}\right)$ generated by (the homotopy class of) $f$. We prove

Theorem A. If $f, g: S^{m-1} \rightarrow \bigvee S^{n}$ represent suspension elements of finite order in $\pi_{m-1}\left(\bigvee S^{n}\right)$, then the following are equivalent:

(1) $C_{f}$ and $C_{g}$ are of the same genus.

(2) $G_{f}=G_{g}$.

Combining Theorems 0.2 and A completes the generalisation of Theorem 0.1 to the following more general setting.

Theorem 0.3. If $f, g: S^{m-1} \rightarrow \bigvee S^{n}$ represent suspension elements of finite order in $\pi_{m-1}\left(S^{n}\right)$, then the following are equivalent:

(1) $C_{f}$ and $C_{g}$ are of the same genus.

(2) $C_{f} \vee \bigvee S^{n}$ and $C_{g} \vee \vee S^{n}$ are homotopy equivalent.

(3) $G_{f}=G_{g}$.

(4) $C_{f} \vee W$ and $C_{g} \vee W$ are homotopy equivalent for some finite wedge of spheres $W$.

Thus the torsion subgroup, $T$, of $\pi_{m-1}\left(S^{n}\right)$ places constraints on the genus of $C_{f}$. It follows, for example, that if $T$ is cyclic of order 2 , then there are precisely two possible genera of mapping cones $C_{f}$ for $f: S^{m-1} \rightarrow \bigvee S^{n}$ representing a suspension map of finite order in $\pi_{m-1}\left(\bigvee S^{n}\right)$. Moreover, each genus is trivial, as can readily be seen from the lattice of subgroups of $\mathbf{Z} / 2 \mathbf{Z}$. We can, in fact, say more. We prove that when the number of $n$-cells in $C_{f}$ is large enough, then the genus of $C_{f}$ becomes trivial. More precisely,

Theorem B. Let $f, g: S^{m-1} \rightarrow \bigvee S^{n}$ represent suspension elements of finite order in $\pi_{m-1}\left(\bigvee S^{n}\right)$. Suppose that $T$, the torsion subgroup of $\pi_{m-1}\left(S^{n}\right)$, can be generated by $N$ elements. If $K \geq 2 N$, then $C_{f}$ and $C_{g}$ are of the same genus if and only if they are homotopy equivalent.

Thus we see that the genus of $C_{f}$ is trivial if $K$ is large enough in the cases under consideration. This stands in stark contrast to the situation which obtains when $f$ represents an element of infinite order and the Hilton-Hopf form has maximum rank (cf. [1]): if, for example, $C_{f}$ is a $(2 r-1)$-connected differentiable $4 r$-manifold, then the genus only becomes nontrivial when the number of cells in dimension $2 r$ is large enough (cf. [5]).

The rest of this note is divided into a number of sections. Section 1 serves to fix notation and recall background. Section 2 contains the definition of $G_{f}$ and the proof of Theorem A. Section 3 contains the proof of Theorem B. The final section contains comments on the results.

\section{Preliminaries}

We use $\simeq$ to denote homotopy equivalence, and write $A \sim B$ when $A$ and $B$ are of the same genus. For the rest of this note we choose, once and for all, 
integers $m>n>1$ and $K \geq 1$. We write all groups additively and write $T$ for the torsion subgroup of $\pi_{m-1}\left(S^{n}\right)$. We use $t$ and $N$ to denote respectively the exponent of $T$ and the minimum number of generators of $T$. When we need $T$ explicitly, we write it as $\mathbf{Z} / t_{1} \mathbf{Z} \oplus \cdots \oplus \mathbf{Z} / t_{N} \mathbf{Z}$, where $\mathbf{Z} / t_{i} \mathbf{Z}$ is generated by $u_{i}(i=1, \ldots, N)$.

The spaces we study are mapping cones of maps $f: S^{m-1} \rightarrow \bigvee S^{n}$. Homotopy classes of maps between such spaces are classified by homotopy classes of homotopy commutative diagrams

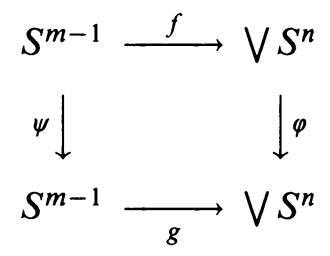

Such diagrams admit an algebraic characterisation (cf. [1]). If $f: S^{m-1} \rightarrow \bigvee S^{n}$ represents a suspension element of finite order in $\pi_{m-1}\left(\bigvee S^{n}\right)$, then we can represent (the homotopy class of) $f$ by $\underline{x}(f)$, which is a column $K$-"vector" each of whose coefficients is a suspension element of $T$. In fact we can write

$$
\underline{x}(f)=\left[\begin{array}{c}
f_{1} \\
\vdots \\
f_{K}
\end{array}\right],
$$

where $f_{i}=q_{i} \circ f$. Then the diagram above corresponds to the equation

$$
\operatorname{deg}(\psi) \underline{x}(g)=\underline{A}(\varphi) \underline{x}(f),
$$

where $\operatorname{deg}(\psi)$ is the degree of the self-map $\psi$ of $S^{m-1}$ and $\underline{A}(\varphi)$ is the $K \times K$ integral matrix whose $(i, j)$ th coefficient is the degree of $i n_{i} \circ \varphi \circ q_{j}$. Here $i n_{i}$ denotes the $i$ th canonical inclusion in the coproduct and $q_{j}$ the $j$ th collapsing map, which acts as the identity map on the $j$ th summand and as the constant map elsewhere. We recall that $q_{i} \circ i n_{i}$ is (homotopic to) the identity map on $S^{n}$, that $q_{i} \circ i n_{j}$ is the constant map if $i \neq j$, and that $\sum i n_{i} \circ q_{i}$ is (homotopic to) the identity map on $\bigvee S^{n}$.

The equation above expresses the facts that because composition is always right additive, $\underline{x}(g \circ \psi)=\operatorname{deg}(\psi) \underline{x}(g)$, and that in our case $\underline{x}(\varphi \circ f)=\underline{A}(\varphi) \underline{x}(f)$ since composition is also left additive when $f$ is a suspension element.

Furthermore, the map between $C_{f}$ and $C_{g}$ determined by the above diagram is a $p$-equivalence (respectively a homotopy equivalence) if and only if $\operatorname{deg}(\psi)$ and $\operatorname{det}(\underline{A}(\varphi))$ are both coprime to $p$ (respectively $= \pm 1$ ). This allows us to characterise the genus of $C_{f}$ for $f$ representing a suspension element of finite order.

Lemma 1.1. If $f, g: S^{m-1} \rightarrow \bigvee S^{n}$ represent suspension elements of finite order, then $C_{f}$ and $C_{g}$ are of the same genus if and only if there are $\psi$ and $\varphi$ as in the above diagram such that both $\operatorname{deg}(\psi)$ and $\operatorname{det}(\underline{A}(\varphi))$ are coprime to $t$, the exponent of $T$.

When we need to appeal to the stucture of $T$ as $\mathbf{Z} / t_{1} \mathbf{Z} \oplus, \ldots, \oplus \mathbf{Z} / t_{N} \mathbf{Z}$, 
we write $f_{i}$ as $\left(f_{i 1}, \ldots, f_{i N}\right)$ and $\underline{x}(f)$ as

$$
\left[\begin{array}{ccc}
f_{11} & \cdots & f_{1 N} \\
\vdots & & \vdots \\
f_{K 1} & \cdots & f_{K N}
\end{array}\right]
$$

where each $f_{i j}=l_{i j} u_{j}$ for some $l_{i j} \in\left\{0, \ldots, t_{j}-1\right\}$. In fact, it may be assumed without loss of generality that this "matrix" is in "upper triangular" form-that is, $f_{i j}=0$ whenever $i>j$-as is shown in the next lemma.

Lemma 1.2. Let $f: S^{m-1} \rightarrow \bigvee S^{n}$ represent a suspension element of finite order in $\pi_{m-1}\left(\bigvee S^{n}\right)$. Then $C_{f}$ is homotopy equivalent to $C_{\bar{f}}$ for some $\bar{f}: S^{m-1} \rightarrow$ $\bigvee S^{n}$ with $f_{i j}=0$ for $i>j$.

Proof. We reduce the matrix of $f$ to echelon form by means of a suitable invertible integral matrix $A$ and observe that any $K \times K$ integral matrix can be realised by a self-map of $\bigvee S^{n}$-that is, for each $A$ there is a map $\varphi: \bigvee S^{n} \rightarrow$ $\bigvee S^{n}$ with $\underline{A}(\varphi)=A$. If $\varphi$ realises $A$, then putting $\bar{f}:=\varphi \circ f$ we see that $\underline{x}(\bar{f})$ is of the required form and that $\varphi$ together with the identity map of $S^{m-1}$ determine a homotopy equivalence $C_{f} \simeq C_{\bar{f}}$.

Next we introduce the group $G_{f}$ and prove the results announced in the introduction.

\section{THE GROUP $G_{f}$}

Any map $f: S^{m-1} \rightarrow \bigvee S^{n}$ determines $K$ maps $f_{i}: S^{m-1} \rightarrow S^{n}$. Theymore accurately, their homotopy classes-generate a subgroup of $\pi_{m-1}\left(S^{n}\right)$, which is to be the focus of attention in this section. Formally,

Definition. Given a map $f: S^{m-1} \rightarrow \bigvee S^{n}$, the group $G_{f}$ denotes the subgroup of $\pi_{m-1}\left(S^{n}\right)$ generated by $\left\{f_{i} \mid i=1, \ldots, K\right\}$, where $f_{i}:=q_{i} \circ f$.

Lemma 2.1. If $f: S^{m-1} \rightarrow \bigvee S^{n}$ represents a suspension element in $\pi_{m-1}\left(\bigvee S^{n}\right)$, then $G_{f}$ depends only on the homotopy type of $C_{f}$.

Proof. $C_{f} \simeq C_{g}$ if and only if $\underline{x}(g)=A \underline{x}(f)$ for some suitable matrix $A \in$ $\mathbf{G L}(K, \mathbf{Z})$. But then each $g_{i}$ is an integral linear combination of $\left\{f_{1}, \ldots, f_{K}\right\}$. Thus $G_{g} \subseteq G_{f}$.

The reverse inclusion follows by symmetry.

As a partial converse to Lemma 2.1, we see that a choice of generators of $G_{f}$ determines the homotopy type of $C_{f}$, as we now verify.

Lemma 2.2. Take $h_{i}: S^{m-1} \rightarrow S^{n}(i=1, \ldots, K)$ which represent suspension elements in $\pi_{m-1}\left(S^{n}\right)$. Then there is an $f: S^{m-1} \rightarrow \bigvee S^{n}$, unique up to homotopy, such that $f_{i} \simeq h_{i}$.

Proof. Take any $f: S^{m-1} \rightarrow \bigvee S^{n}$ representing a suspension element. Then

$$
f=\mathrm{id} \circ f=\left(\sum_{i=1}^{K} i n_{i} \circ q_{i}\right) \circ f=\sum_{i=1}^{K} i n_{i} \circ q_{i} \circ f=\sum_{i=1}^{K} i n_{i} \circ f_{i} .
$$

(The third equality holds because $f$ is a suspension element.) Thus the $f_{i}$ determine $f$ (up to homotopy). Given $h_{i}(i=1, \ldots, K)$, put $f:=$ $\sum_{i=1}^{K} i n_{i} \circ h_{i}$ and the result follows. 
However, the group $G_{f}$ does not itself determine the homotopy type of $C_{f}$. This is already true when $K=1$, which is one of the main results of [6]. Nevertheless, $G_{f}$ does determine the genus of $C_{f}$, as we now prove.

Theorem 2.3 (Theorem A). Take $f, g: S^{m-1} \rightarrow \bigvee S^{n}$ representing suspension elements of finite order in $\pi_{m-1}\left(\bigvee S^{n}\right)$. Then $C_{f}$ and $C_{g}$ are of the same genus if and only if $G_{f}=G_{g}$.

Proof. Suppose that $C_{f}$ and $C_{g}$ are of the same genus. Then there are selfmaps $\psi$ of $S^{m-1}$ and $\varphi$ of $\bigvee S^{n}$ such that

$$
\operatorname{deg}(\psi) \underline{x}(g)=\underline{A}(\varphi) \underline{x}(f)
$$

with both $\operatorname{deg}(\psi)$ and $\operatorname{det}(\underline{A}(\varphi))$ coprime to $t$. Choose $d$ such that $d \cdot \operatorname{deg}(\psi)$ $\equiv 1(\bmod t)$. Put $A:=d \underline{A}(\varphi)$. Then $\underline{x}(f)=A \underline{x}(g)$, and $G_{f}=G_{g}$ follows as in Lemma 2.1.

For the converse, suppose that $G_{f}=G_{g}$, so that we can write each $g_{j}$ as an integral linear combination of the $f_{i}$ and vice versa. Thus we can find a $K \times K$ integral matrix $A$, whose determinant is coprime to $t$ such that

$$
\underline{x}(g)=A \underline{x}(f) .
$$

Now choose $\varphi: \bigvee S^{n} \rightarrow \bigvee S^{n}$ with $\underline{A}(\varphi)=A$, and put $\psi:=\operatorname{id}_{S^{m-1}}$. By Lemma 1.1, $C_{f} \sim C_{g}$.

It follows from this theorem that the lattice of subgroups of $T$ classifies the homotopy types of mapping cones $C_{f}$ into their respective genera when $f: S^{m-1} \rightarrow \bigvee S^{n}$ represents a suspension element of finite order. Thus, for example, if there are more summands in $\bigvee S^{n}$ than the minimum number of generators of $G_{f}$, then $C_{f}$ is $\vee$-decomposable. If there are even more summands in $\bigvee S^{n}$, then the genus of $C_{f}$ consists of only a single homotopy type. We prove these statements in the next section.

\section{The genUS OF $C_{f}$ FOR LARGe $K$}

We begin by noting that if $K$ is sufficiently large, then the space $C_{f}$ is decomposable with respect to $V$-sum. More precisely,

Lemma 3.1. If $K>N$, then $C_{f}$ is homotopy equivalent to $C_{f} \vee \bigvee^{K-N} S^{n}$ for some $\bar{f}: S^{m-1} \rightarrow \bigvee^{N} S^{n}$.

Proof. By Lemma 1.2, we may assume that $f_{i j}=0$ for $i>j$. Let

$$
q: \bigvee_{i=1}^{K} S^{n} \rightarrow \bigvee_{i=1}^{N} S^{n}
$$

be the collapsing map onto the first $N$ summands. Then $\bar{f}:=q \circ f$ has the required properties, since $f_{i j}=0$ for every $i>N$.

We observe that while we have made a canonical choice of $\bar{f}$, once the direct sum representation of $T$ has been fixed, $C_{f}$ is determined only up to genus by the condition in the statement of the lemma (cf. [3]).

We can now apply Theorems 0.3 and 3.1 to prove Theorem B of the introduction; we show that the genus of $C_{f}$ is trivial whenever $K$ is at least $2 N$. 
Theorem 3.2. Take $f, g: S^{m-1} \rightarrow \bigvee S^{n}$ representing suspension elements of finite order in $\pi_{m-1}\left(S^{n}\right)$. If $K \geq 2 N$, then $C_{f}$ and $C_{g}$ are of the same genus if and only if they are homotopy equivalent.

Proof. Clearly only the "only if" part requires proof.

Take $f, g: S^{m-1} \rightarrow \bigvee S^{n}$ as in the statement of the theorem. Suppose $K \geq 2 N$ and $C_{f} \sim C_{g}$. By Lemma 3.1, we can find $\bar{f}, \bar{g}: S^{m-1} \rightarrow \bigvee_{1}^{K} S^{n}$ such that $C_{f} \simeq C_{\bar{f}} \vee \bigvee^{K-N} S^{n}$ and $C_{g} \simeq C_{\bar{g}} \vee \bigvee^{K-N} S^{n}$. But then $C_{\bar{f}} \vee \bigvee^{K-N} S^{n}$ is of the same genus as $C_{\bar{g}} \vee \bigvee^{K-N} S^{n}$. Hence, by Theorem 0.3, $C_{\bar{f}} \vee \bigvee^{2 K-N} S^{n} \simeq$ $C_{\tilde{g}} \vee \bigvee^{2 K-N} S^{n}$. Applying Theorem 0.3 once again, we find that $C_{\bar{f}}$ and $C_{\tilde{g}}$ are of the same genus, so that a final appeal to Theorem 0.3 allows us to conclude that $C_{\bar{f}} \vee \bigvee^{N} S^{n} \simeq C_{\tilde{g}} \vee \bigvee^{N} S^{n}$. But then $C_{\bar{f}} \vee \bigvee^{K-N} S^{n}$ is homotopy equivalent to $C_{\tilde{g}} \vee \bigvee^{K-N} S^{n}$, as $K-N \geq N$.

Combining this result with Theorem 2.3, we obtain the following converse to Lemma 2.1.

Corollary 3.3. If $K \geq 2 N$, then $G_{f}$ determines the homotopy type of $C_{f}$.

Observe that the condition $K \geq 2 N$ cannot be relaxed in general, as was shown in [6] for the case $K=1$.

Remarks. The results presented in this note can be improved without modification of the arguments presented. It is, for example, not necessary to assume that the maps under consideration represent suspension elements. It would be sufficient to require that their Hilton-Hopf invariants all vanish, for then composition is once again both right and left additive.

The assumption of finite order is also not necessary. We could have argued formally exactly as above had we chosen to introduce the convention that $\mathbf{Z} / \infty \mathbf{Z}$ is just $\mathbf{Z}$ and that the only integers coprime to $\infty$ are \pm 1 . Where we used the finiteness of $\pi_{m-1}\left(S^{n}\right)$ it would have sufficed to use Noetherianness to draw the same conclusions. Since $\pi_{m-1}\left(S^{n}\right)$ is cyclic whenever it has a suspension element of infinite order, we could have drawn the conclusion that $C_{f} \simeq C_{g}$ whenever $C_{f} \sim C_{g}$ in such a case, for each $G_{f}$ has precisely two distinct possible generators, each the negative of the other. The only case in which suspension elements of infinite order arise is when $m=n+1$. In this case the mapping cones are simply connected Moore spaces, and we would simply have drawn the reassuring conclusion that a simply connected Moore space is determined up to homotopy equivalence by its single nontrivial ordinary reduced homology group. Hence the greater generality would have been more apparent than real, and at best, of academic interest.

A better estimate for the number of $n$-cells needed to ensure that the genus of $C_{f}$ be trivial could be obtained by arguing-mutatis mutandis-with $G_{f}$ in lieu of $T$.

It was felt that these marginal improvements did not warrant the more elliptic language required and that the bound we have given for $K$ has the advantage of being independent of the particular $C_{f}$ chosen.

Finally we note that the full force of Theorem 0.3 was not needed in the proof of Theorem 3.2, since the group $G_{f}$ did not appear at any stage. However it was the realisation that $G_{f}$ characterises the genus of $C_{f}$ which invited the conjecture that the minimum number of generators of $T$ must constrain the 
size of the genus set. Llerena's results-quoted here as Theorem 0.2 -provided the means to verify this.

\section{BIBLIOGRAPHY}

1. I. Bokor, On genus and cancellation in homotopy, Israel J. Math. 73 (1991), 361-379.

2. P. Hilton, G. Mislin, and J. Roitberg, Localization of nilpotent groups and spaces, NorthHolland, Amsterdam, 1975.

3. I. Llerena, Wedge cancellation and genus (submitted).

4. __ Wedge cancellation of certain mapping cones, Compositio Math. 81 (1992), 1-17.

5. J. Milnor, On simply connected 4-manifolds, Proc. Internat. Sympos. Algebraic Topology, Univ. of Mexico, Universidad Nacional Autónoma de México and UNESCO, 1958, pp. 122-128.

6. E. A. Molnar, Relation between wedge cancellation and localization for complexes with two cells, J. Pure Appl. Algebra 3 (1972), 77-81.

7. C. Wilkerson, Genus and cancellation, Topology 14 (1975), 29-36.

75 Mount St., Coogee 2034, New South Wales, Australia

Current address: Department of Mathematics, Statistics and Computer Science, University of New England, Armidale 2351, New South Wales, Australia 